Acta Crystallographica Section E

Structure Reports

Online

ISSN 1600-5368

\section{David J. Watkin, ${ }^{\mathrm{a} *}$ Loren L.} Parry, ${ }^{a}$ Raquel Soengas, ${ }^{\text {b }}$ Ken Izumoric $^{\mathrm{c}}$ and George W. J. Fleet ${ }^{\mathrm{b}}$

${ }^{a}$ Chemical Crystallography, Chemistry Research Laboratory, University of Oxford, Oxford OX1 3TA, England, bepartment of Organic Chemistry, Chemical Research Laboratory, Oxford University, Mansfield Road, Oxford OX1

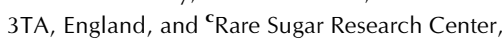
Kagawa University, Mikicho, Kagawa

761-0795, Japan

Correspondence e-mail:

david.watkin@chem.ox.ac.uk

\section{Key indicators}

Single-crystal X-ray study

$T=190 \mathrm{~K}$

Mean $\sigma(\mathrm{C}-\mathrm{C})=0.004 \AA$

$R$ factor $=0.049$

$w R$ factor $=0.097$

Data-to-parameter ratio $=11.6$

For details of how these key indicators were automatically derived from the article, see http://journals.iucr.org/e.

\title{
2-C-Benzyloxymethyl-2,3:5,6-di-O-iso- propylidene-D-allono-1,4-lactone
}

The crystal structure of the title benzyl ether, $\mathrm{C}_{20} \mathrm{H}_{26} \mathrm{O}_{7}$, establishes the stereochemistry of the major diacetonide product from the Kiliani reaction of D-psicose. There are two independent molecules in the cell related by a pseudo-twofold screw axis. There are no suitable donors for strong hydrogen bonds: the molecules are simply in van der Waals contact.

\section{Comment}

Almost all of the readily available carbohydrate building blocks have linear carbon chains (Bols, 1996; Lichtenthaler \& Peters, 2004). However, the Kiliani reaction on ketohexoses provides branched sugar lactones bearing a hydroxymethyl substituent at C-2. Acetonation of the crude mixtures of the resulting lactones gives reasonable yields of crystalline diacetonides (Hotchkiss et al., 2004) which are suitable chiral starting materials for the synthesis of branched sugar mimics (Simone et al., 2005). Ambiguities in the structures of the acetonides arise from (i) the stereochemistry at the quaternary carbon and (ii) the ring size of the ketal protecting groups. The structures of diacetonides derived from three of the four diastereomeric ketohexoses [D-fructose (Cowley et al., 2004; van Ameijde et al., 2004), L-sorbose (Anderson et al., 1977) and D-tagatose (Harding et al., 2005; Shallard-Brown et al., 2004)] have been firmly established by X-ray crystallographic analysis. The fourth diastereomeric ketohexose D-psicose (1) is available from equilibration of D-fructose by D-tagatose 3epimerase (Granstrom et al., 2004; Izumori, 2002; Takeshita et al., 2000; Itoh \& Izumori, 1996).

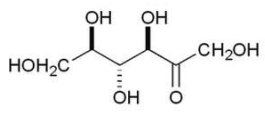

(1)

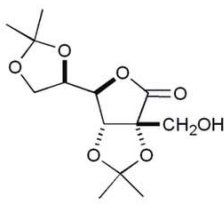

(2)
Received 10 August 2005 Accepted 12 August 2005 Online 17 August 2005

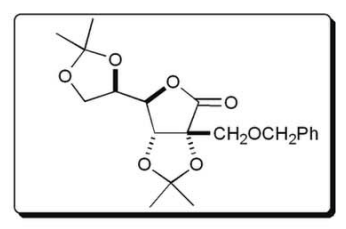

(3)
The sequential treatment of D-psicose (1) with sodium cyanide, followed by extraction of the crude lactones with acetone in the presence of sulfuric acid gave, as the major product (Soengas et al., 2005), a diacetonide tentatively assigned as structure (2); although the cis-fused diacetonide (2) was easily crystallized, the crystals were not suitable for Xray crystallographic analysis. However, reaction of (2) with benzyl bromide and sodium hydride in the presence of tetra-nbutylammonium iodide in dimethylformamide afforded the corresponding benzyl ether (3), which formed crystals for X;ray suitable analysis. This paper reports the crystal structure of (3) (Fig. 1) which unequivocally establishes the stereo- 


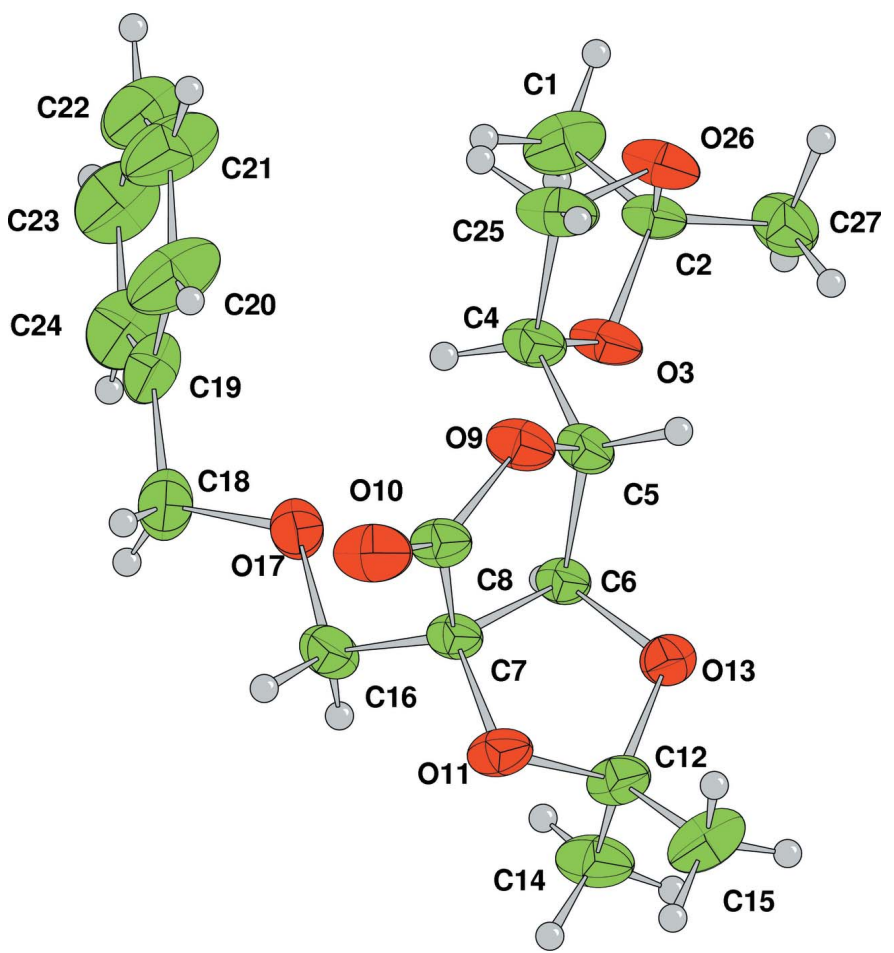

Figure 1

View of one of the two independent molecules in the asymmetric unit, with atomic displacement ellipsoids drawn at the $50 \%$ probability level. $\mathrm{H}$-atom radii are arbitary.

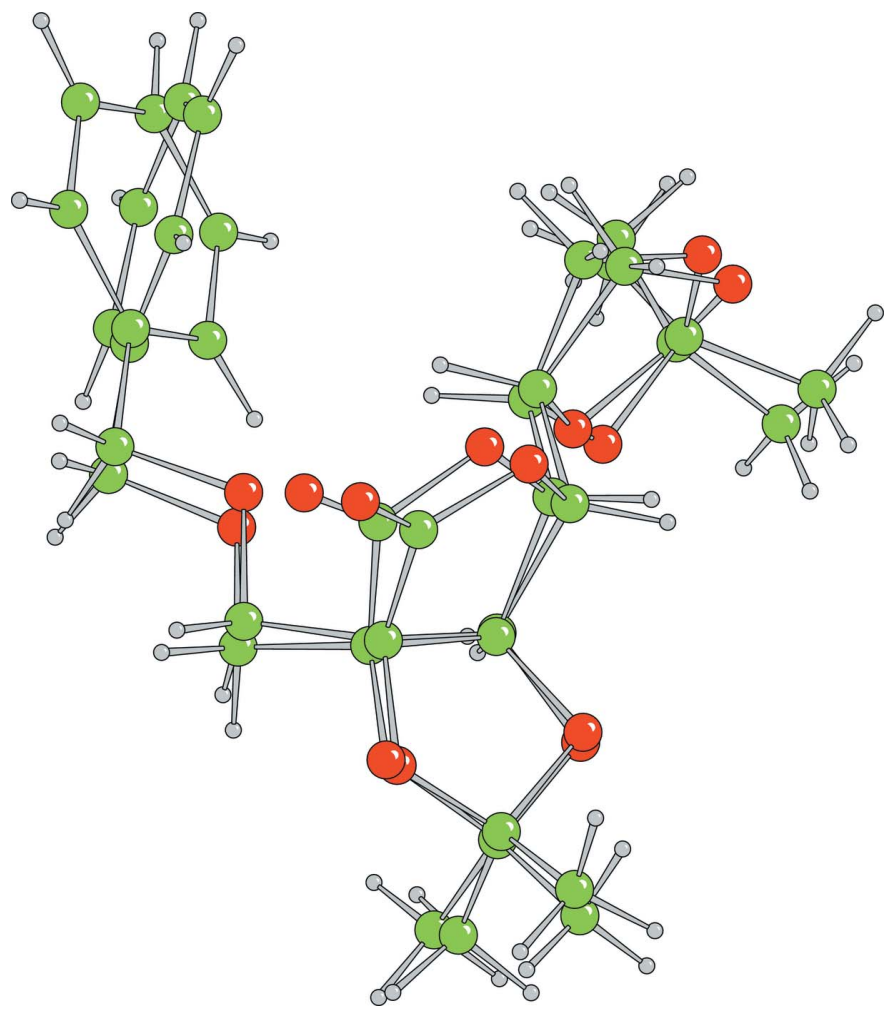

Figure 2

View of the two independent molecules superimposed to give the leastsquares best fit between the atom coordinates.

chemistry of the major lactone product (2) from the Kilianiacetonation sequence on D-psicose.

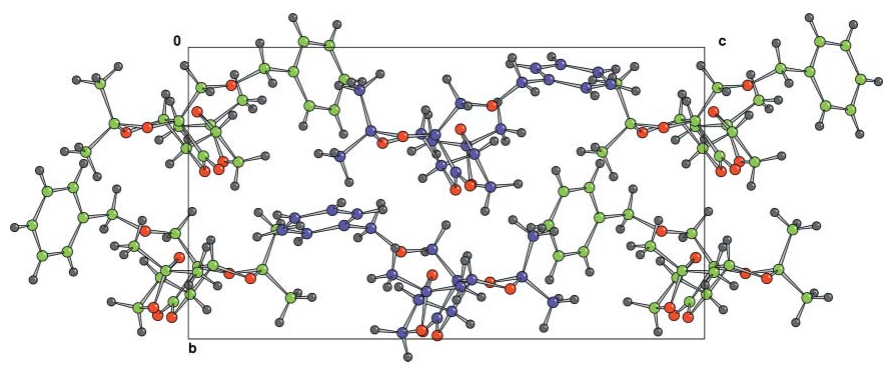

Figure 3

An $a$ axis projection of the title compound. The $\mathrm{C}$ atoms in one of the independent molecules are coloured green, those in the other blue.

The crystals were in the form of large fragile prisms which crushed easily. A long sample was eventually selected and mounted on a nylon loop using perfluoropolyether oil so that the damaged end of the crystal was outside the X-ray beam. By mounting the crystal approximately parallel to the $\varphi$ axis, the changes in illuminated volume were kept to a minimum, and were taken into account (Görbitz, 1999) by the multi-scan inter-frame scaling (DENZO/SCALEPACK, Otwinowski \& Minor, 1997).

There are two independent molecules in the asymmetric unit related by a pseudo-twofold screw axis $(0.51-x, 0.46+y$, $1.50-z$ ). The molecules differ mainly in the orientation of the phenyl group (Fig. 2). The structure contains no hydrogen bonds and consists of layers loosely packed perpendicular to the $c$ axis (Fig. 3). Alternate layers contain only molecules of one type.

\section{Experimental}

The benzyl ether (3) was crystallized from ethyl acetate-cyclohexane (m.p. $345-346 \mathrm{~K}) ;[\alpha]_{22}^{\mathrm{D}}-30.0\left(c, 1.06\right.$ in chloroform); $v_{\max }(\mathrm{NaCl})$ : $1781(-\mathrm{C}=\mathrm{O}) \mathrm{cm}-1 ;{ }^{1} \mathrm{H}$ NMR $\left(\mathrm{CDCl}_{3}\right): \delta 1.27,1.40,1.46[3 \times s, 12 \mathrm{H}$, $\left.2 \times-\mathrm{C}\left(\mathrm{CH}_{3}\right)_{2}\right], 3.73\left(d, 1 \mathrm{H}, J_{2^{\prime}, 2^{\prime \prime}} 9.0 \mathrm{~Hz}, \mathrm{H} 2^{\prime}\right), 3.92-3.97(m, 3 \mathrm{H}, \mathrm{H} 6$, H6? , H4), 4.12-4.15 ( $m, 1 \mathrm{H}, \mathrm{H} 5), 4.29$ (d, 1H, $\left.J_{2^{\prime}, 2^{\prime \prime}} 9.0 \mathrm{~Hz}, \mathrm{H} 2^{\prime \prime}\right), 4.57$ $\left(s, 2 \mathrm{H},-\mathrm{OCH}_{2} \mathrm{Ph}\right), 4.85(s, 1 \mathrm{H}, \mathrm{H} 3) ;{ }^{13} \mathrm{C} \mathrm{NMR}\left(\mathrm{CDCl}_{3}\right): \delta 24.60,26.07$, 26.60, $26.63\left[2 \times-\mathrm{C}\left(\mathrm{CH}_{3}\right)_{2}\right], 66.47$ (C2'), 69.34 (C6), 73.37 (C5), 73.99 $\left(-\mathrm{OCH}_{2} \mathrm{Ph}\right), 80.05(\mathrm{C} 3), 83.13(\mathrm{C} 4), 83.70(\mathrm{C} 2), 109.91,113.33[2 \times$ $\left.-\mathrm{C}\left(\mathrm{CH}_{3}\right)_{2}\right], 127.76,127.91,128.29$ (-CHAr), 136.70 (-CAr) 174.56 $(\mathrm{C} 1) ; m / z\left(\mathrm{NH}_{3}, \mathrm{ES}+\right): 401(M+\mathrm{Na})^{+}$.

\section{Crystal data}

$\mathrm{C}_{20} \mathrm{H}_{26} \mathrm{O}_{7}$
$M_{r}=378.42$

Monoclinic, $P 2_{1}$

$a=10.3839$ (2) ^

$b=10.4574(2) \AA$

$c=19.0310(5) \AA$

$\beta=103.8061(9)^{\circ}$

$V=2006.84(8) \AA^{3}$

$Z=4$

Data collection

Nonius Kappa CCD diffractometer $\omega$ scans

Absorption correction: multi-scan (DENZO/SCALEPACK

Otwinowski \& Minor, 1997)

$T_{\min }=0.81, T_{\max }=0.96$

11499 measured reflections

\author{
$D_{x}=1.252 \mathrm{Mg} \mathrm{m}^{-3}$ \\ Mo $K \alpha$ radiation \\ Cell parameters from 3433 \\ reflections \\ $\theta=1-30^{\circ}$ \\ $\mu=0.09 \mathrm{~mm}^{-1}$ \\ $T=190 \mathrm{~K}$ \\ Prism, colourless \\ $1.20 \times 0.75 \times 0.45 \mathrm{~mm}$
}




\section{Refinement}

Refinement on $F^{2}$ $R\left[F^{2}>2 \sigma\left(F^{2}\right)\right]=0.049$

$w R\left(F^{2}\right)=0.097$

$S=0.95$

5635 reflections

487 parameters

$\mathrm{H}$-atom parameters constrained

$$
\begin{aligned}
& w=1 /\left[\sigma^{2}\left(F^{2}\right)+(0.03 P)^{2}\right. \\
& \quad+0.6 P] \\
& \quad \text { where } P=\left[\max \left(F_{\mathrm{o}}{ }^{2}, 0\right)+2 F_{\mathrm{c}}{ }^{2}\right] / 3 \\
& (\Delta / \sigma)_{\max }=0.001 \\
& \Delta \rho_{\max }=0.23 \mathrm{e}^{-3} \\
& \Delta \rho_{\min }=-0.17 \mathrm{e}^{-3}
\end{aligned}
$$

The $\mathrm{H}$ atoms were all located in a difference map, but those attached to $\mathrm{C}$ atoms were repositioned geometrically. The $\mathrm{H}$ atoms were initially refined with soft restraints on the bond lengths and angles to regularize their geometry $(\mathrm{C}-\mathrm{H}=0.93-98 \AA)$ and isotropic displacement parameters $\left[U_{\text {iso }}(\mathrm{H})=1.2-1.5 U_{\text {eq }}(\mathrm{C})\right]$, after which they were refined with riding constraints. In the absence of significant anomalous dispersion effects, Friedel pairs were averaged.

Data collection: COLLECT (Nonius, 2001); cell refinement: DENZO/SCALEPACK; data reduction: DENZO/SCALEPACK (Otwinowski \& Minor, 1997); program(s) used to solve structure: SIR92 (Altomare et al., 1994); program(s) used to refine structure: CRYSTALS (Betteridge et al., 2003); molecular graphics: CAMERON (Watkin et al., 1996); software used to prepare material for publication: CRYSTALS.

Financial support (to $R S$ ) provided by the Xunta de Galicia is gratefully acknowledged.

\section{References}

Altomare, A., Cascarano, G., Giacovazzo, C., Guagliardi, A., Burla, M. C., Polidori, G. \& Camalli, M. (1994). J. Appl. Cryst. 27, 435.
Ameijde, J. van, Cowley, A. R., Fleet, G. W. J., Nash, R. J, Simone, M. I. \& Soengas, R. (2004). Acta Cryst. E60, o2140-o2141.

Anderson, R. A., Einstein, F. W. B., Hoge, R. \& Slessor, K. N. (1977). Acta Cryst. B33, 2780-2783.

Betteridge, P. W., Carruthers, J. R., Cooper, R. I., Prout, K. \& Watkin, D. J. (2003). J. Appl. Cryst. 36, 1487.

Bols, M. (1996). Carbohydrate Building Blocks. New York: John Wiley \& Sons.

Cowley, A. R., Fleet, G. W. J., Simone, M. I. \& Soengas, R. (2004). Acta Cryst. E60, o2142-o2143.

Görbitz, C. H. (1999). Acta Cryst. B55, 1090-1098.

Granstrom, T. B., Takata, G., Tokuda, M. \& Izumori, K. (2004). J. Biosci. Bioeng. 97, 89-94.

Harding, C. C., Watkin, D. J., Cowley, A. R., Soengas, R., Skytte, U. P. \& Fleet, G. W. J. (2005). Acta Cryst. E61, o250-0252.

Hotchkiss, D., Soengas, R., Simone, M. I., van Ameijde, J., Hunter, S., Cowley, A. R. \& Fleet, G. W. J. (2004). Tetrahedron Lett. 45, 94619464.

Itoh, H. \& Izumori, K. (1996). J. Ferment. Bioeng. 81, 351-353.

Izumori, K. (2002). Naturwissenschaften, 89, 120-124.

Lichtenthaler, F. W. \& Peters, S. (2004). Compt. Rend. Chim. 7, 6590.

Nonius (2001). COLLECT. Nonius BV, Delft, The Netherlands.

Otwinowski, Z. \& Minor, W. (1997). Methods in Enzymology, Vol. 276, Macromolecular Crystallography, Part A, edited by C. W. Carter Jr \& R. M. Sweet, pp. 307-326. New York: Academic Press.

Shallard-Brown, H. A., Harding, C. C., Watkin, D. J., Soengas, R., Skytte, U. P. \& Fleet G. W. J. (2004). Acta Cryst. E60, o2163-o2164.

Sheldrick, G. M. (1997). SHELXL97. University of Göttingen, Germany.

Simone, M. I., Soengas, R., Newton, C. R., Watkin, D. J. \& Fleet, G. W. J. (2005). Tetrahedron Lett. 46, 5761-5765.

Soengas, R., Izumori, K., Simone, M. I., Watkin, D. J., Skytte, U. P., Soetaert, W. \& Fleet, G. W. J. (2005). Tetrahedron Lett. 46, 5755-5759.

Takeshita, K., Suga, A., Takada, G. \& Izumori, K. (2000). J. Biosci. Bioeng. 90, 453-455.

Watkin, D. J., Prout, C. K. \& Pearce, L. J. (1996). CAMERON. Chemical Crystallography Laboratory, Oxford, England. 


\section{supporting information}

Acta Cryst. (2005). E61, o2955-o2957 [https://doi.org/10.1107/S1600536805025791]

\section{2-C-Benzyloxymethyl-2,3:5,6-di-O-isopropylidene-D-allono-1,4-lactone}

David J. Watkin, Loren L. Parry, Raquel Soengas, Ken Izumori and George W. J. Fleet

2-C-Benzyloxymethyl-2,3:5,6-di-O-isopropylidene-D-allono-1,4-lactone

Crystal data

$\mathrm{C}_{20} \mathrm{H}_{26} \mathrm{O}_{7}$

$M_{r}=378.42$

Monoclinic, $P 2_{1}$

Hall symbol: P 2yb

$a=10.3839$ (2) $\AA$

$b=10.4574(2) \AA$

$c=19.0310(5) \AA$

$\beta=103.8061(9)^{\circ}$

$V=2006.84(8) \AA^{3}$

$Z=4$

\section{Data collection}

Nonius Kappa CCD diffractometer

Graphite monochromator

$\omega$ scans

Absorption correction: multi-scan

(DENZO/SCALEPACK; Otwinowski \& Minor, 1997)

$T_{\min }=0.81, T_{\max }=0.96$

Refinement

Refinement on $F^{2}$

Least-squares matrix: full

$R\left[F^{2}>2 \sigma\left(F^{2}\right)\right]=0.049$

$w R\left(F^{2}\right)=0.097$

$S=0.95$

5635 reflections

487 parameters

1 restraint
$F(000)=808$

$D_{\mathrm{x}}=1.252 \mathrm{Mg} \mathrm{m}^{-3}$

Mo $K \alpha$ radiation, $\lambda=0.71073 \AA$

Cell parameters from 3433 reflections

$\theta=1-30^{\circ}$

$\mu=0.09 \mathrm{~mm}^{-1}$

$T=190 \mathrm{~K}$

Prism, colourless

$1.20 \times 0.75 \times 0.45 \mathrm{~mm}$

11499 measured reflections

5664 independent reflections

5635 reflections with $I>-3 \sigma(I)$

$R_{\text {int }}=0.027$

$\theta_{\max }=30.1^{\circ}, \theta_{\min }=1.1^{\circ}$

$h=-14 \rightarrow 14$

$k=-7 \rightarrow 14$

$l=-26 \rightarrow 26$

Primary atom site location: structure-invariant direct methods

Hydrogen site location: inferred from neighbouring sites

$\mathrm{H}$-atom parameters constrained

$w=1 /\left[\sigma^{2}\left(F^{2}\right)+(0.03 P)^{2}+0.6 P\right]$

where $P=\left[\max \left(F_{\mathrm{o}}{ }^{2}, 0\right)+2 F_{\mathrm{c}}{ }^{2}\right] / 3$

$(\Delta / \sigma)_{\max }=0.001$

$\Delta \rho_{\max }=0.23$ e $\AA^{-3}$

$\Delta \rho_{\min }=-0.17$ e $\AA^{-3}$

Fractional atomic coordinates and isotropic or equivalent isotropic displacement parameters $\left(\AA^{2}\right)$

\begin{tabular}{lllll}
\hline & $x$ & $y$ & $z$ & $U_{\text {iso }} * / U_{\text {eq }}$ \\
\hline $\mathrm{C} 1$ & $0.6912(3)$ & $0.6817(3)$ & $0.89496(16)$ & 0.0613 \\
$\mathrm{C} 2$ & $0.6732(2)$ & $0.7661(2)$ & $0.95659(12)$ & 0.0358 \\
$\mathrm{O} 3$ & $0.56299(14)$ & $0.72111(16)$ & $0.98275(10)$ & 0.0429 \\
$\mathrm{C} 4$ & $0.4476(2)$ & $0.7902(2)$ & $0.94647(13)$ & 0.0393
\end{tabular}




\begin{tabular}{|c|c|c|c|c|}
\hline $\mathrm{C} 5$ & $0.3862(2)$ & $0.8461(2)$ & $1.00390(12)$ & 0.0342 \\
\hline C6 & $0.32997(19)$ & $0.74764(19)$ & $1.04783(11)$ & 0.0309 \\
\hline $\mathrm{C} 7$ & $0.17916(19)$ & $0.7523(2)$ & $1.01840(11)$ & 0.0312 \\
\hline $\mathrm{C} 8$ & $0.1574(2)$ & $0.8728(2)$ & $0.97093(12)$ & 0.0351 \\
\hline O9 & $0.27461(15)$ & $0.92551(15)$ & $0.96805(9)$ & 0.0397 \\
\hline $\mathrm{O} 10$ & $0.05341(16)$ & $0.9189(2)$ & $0.94083(10)$ & 0.0526 \\
\hline $\mathrm{O} 11$ & $0.12530(14)$ & $0.77517(17)$ & $1.07923(8)$ & 0.0389 \\
\hline H11 & 0.7692 & 0.7111 & 0.8752 & $0.1072 *$ \\
\hline $\mathrm{C} 12$ & $0.2311(2)$ & $0.7618(2)$ & $1.14340(12)$ & 0.0385 \\
\hline H12 & 0.7122 & 0.5935 & 0.9107 & $0.1072 *$ \\
\hline $\mathrm{O} 13$ & $0.34809(15)$ & $0.79243(17)$ & $1.12045(8)$ & 0.0389 \\
\hline H13 & 0.6153 & 0.6811 & 0.8572 & $0.1070^{*}$ \\
\hline $\mathrm{C} 14$ & $0.2355(3)$ & $0.6250(3)$ & $1.17066(15)$ & 0.0499 \\
\hline $\mathrm{C} 15$ & $0.2127(3)$ & $0.8590(3)$ & $1.19836(15)$ & 0.0595 \\
\hline $\mathrm{C} 16$ & $0.1155(2)$ & $0.6383(2)$ & $0.97486(13)$ & 0.0409 \\
\hline $\mathrm{O} 17$ & $0.17799(16)$ & $0.63022(18)$ & $0.91661(9)$ & 0.0475 \\
\hline C18 & $0.1019(3)$ & $0.5727(3)$ & $0.85246(14)$ & 0.0523 \\
\hline C19 & $0.1805(3)$ & $0.5838(2)$ & $0.79633(13)$ & 0.0493 \\
\hline $\mathrm{C} 20$ & 0.1947 (4) & $0.7012(3)$ & $0.76556(17)$ & 0.0745 \\
\hline $\mathrm{C} 21$ & $0.2743(5)$ & $0.7150(4)$ & $0.71718(19)$ & 0.0858 \\
\hline $\mathrm{C} 22$ & $0.3377(4)$ & $0.6098(4)$ & $0.69765(17)$ & 0.0723 \\
\hline $\mathrm{C} 23$ & $0.3234(4)$ & $0.4942(3)$ & $0.72696(19)$ & 0.0731 \\
\hline $\mathrm{C} 24$ & $0.2461(3)$ & 0.4807 (3) & $0.77668(17)$ & 0.0645 \\
\hline $\mathrm{C} 25$ & $0.5008(2)$ & $0.8934(3)$ & $0.90304(16)$ & 0.0541 \\
\hline $\mathrm{O} 26$ & $0.63821(16)$ & $0.89243(16)$ & $0.93251(10)$ & 0.0468 \\
\hline $\mathrm{C} 27$ & $0.7940(2)$ & $0.7712(3)$ & $1.01804(15)$ & 0.0502 \\
\hline $\mathrm{H} 41$ & 0.3846 & 0.7327 & 0.9142 & $0.0519^{*}$ \\
\hline H51 & 0.4571 & 0.8998 & 1.0360 & $0.0470^{*}$ \\
\hline H61 & 0.3665 & 0.6607 & 1.0437 & $0.0466^{*}$ \\
\hline C101 & $-0.1863(3)$ & $0.2800(3)$ & $0.60522(15)$ & 0.0542 \\
\hline C102 & $-0.1537(2)$ & $0.3374(2)$ & $0.53881(12)$ & 0.0337 \\
\hline $\mathrm{O} 103$ & $-0.03441(14)$ & $0.28084(15)$ & $0.52740(10)$ & 0.0430 \\
\hline C104 & $0.0740(2)$ & $0.3658(2)$ & $0.55386(13)$ & 0.0365 \\
\hline C105 & $0.1294(2)$ & $0.40541(19)$ & $0.48991(13)$ & 0.0371 \\
\hline C106 & $0.18060(19)$ & 0.29669 (19) & $0.45118(11)$ & 0.0303 \\
\hline C107 & $0.33235(19)$ & $0.30000(18)$ & 0.47837 (11) & 0.0296 \\
\hline C108 & $0.3581(2)$ & $0.4284(2)$ & $0.51802(12)$ & 0.0360 \\
\hline O109 & $0.24325(16)$ & $0.48850(14)$ & $0.51772(10)$ & 0.0434 \\
\hline $\mathrm{O} 110$ & $0.46329(17)$ & $0.47463(18)$ & $0.54578(10)$ & 0.0520 \\
\hline O111 & $0.38350(15)$ & $0.30560(16)$ & $0.41557(8)$ & 0.0392 \\
\hline C112 & $0.2743(2)$ & $0.2868(2)$ & $0.35378(12)$ & 0.0404 \\
\hline O113 & $0.16033(17)$ & $0.32835(17)$ & $0.37628(9)$ & 0.0437 \\
\hline $\mathrm{C} 114$ & $0.2634(3)$ & $0.1469(2)$ & 0.33179 (14) & 0.0505 \\
\hline C115 & $0.2938(3)$ & $0.3743(3)$ & $0.29361(16)$ & 0.0636 \\
\hline C116 & $0.3952(2)$ & $0.1928(2)$ & $0.52760(11)$ & 0.0325 \\
\hline O117 & 0.33834 (14) & $0.20092(15)$ & $0.58821(8)$ & 0.0367 \\
\hline $\mathrm{C} 118$ & $0.3999(2)$ & $0.1202(2)$ & $0.64651(11)$ & 0.0358 \\
\hline C119 & $0.3113(2)$ & $0.11102(19)$ & $0.69815(12)$ & 0.0336 \\
\hline
\end{tabular}




\begin{tabular}{|c|c|c|c|c|}
\hline $\mathrm{C} 120$ & $0.3578(2)$ & $0.1389(2)$ & $0.77052(12)$ & 0.0412 \\
\hline C121 & $0.2762(3)$ & $0.1263(3)$ & $0.81823(14)$ & 0.0526 \\
\hline C122 & $0.1479(3)$ & $0.0854(3)$ & $0.79376(16)$ & 0.0584 \\
\hline $\mathrm{C} 123$ & $0.0992(3)$ & $0.0588(3)$ & $0.72118(18)$ & 0.0561 \\
\hline C124 & $0.1804(2)$ & $0.0716(2)$ & $0.67285(14)$ & 0.0436 \\
\hline C125 & $0.0123(2)$ & $0.4789(2)$ & $0.58536(15)$ & 0.0459 \\
\hline $\mathrm{O} 126$ & $-0.12454(15)$ & $0.47056(15)$ & $0.54808(9)$ & 0.0398 \\
\hline $\mathrm{C} 127$ & $-0.2637(2)$ & $0.3203(2)$ & $0.47264(14)$ & 0.0447 \\
\hline H141 & 0.2995 & 0.6122 & 1.2168 & $0.0839 *$ \\
\hline H142 & 0.1494 & 0.6041 & 1.1787 & $0.0838^{*}$ \\
\hline H143 & 0.2526 & 0.5660 & 1.1356 & $0.0836^{*}$ \\
\hline H151 & 0.2850 & 0.8572 & 1.2396 & $0.1017 *$ \\
\hline H152 & 0.1340 & 0.8423 & 1.2121 & $0.1012^{*}$ \\
\hline H153 & 0.2073 & 0.9437 & 1.1765 & $0.1018^{*}$ \\
\hline H161 & 0.1271 & 0.5628 & 1.0073 & $0.0582^{*}$ \\
\hline H162 & 0.0161 & 0.6520 & 0.9578 & $0.0579 *$ \\
\hline H181 & 0.0150 & 0.6230 & 0.8367 & $0.0714^{*}$ \\
\hline H182 & 0.0768 & 0.4857 & 0.8613 & $0.0722 *$ \\
\hline $\mathrm{H} 201$ & 0.1497 & 0.7703 & 0.7774 & $0.0980^{*}$ \\
\hline H211 & 0.2841 & 0.7975 & 0.6995 & $0.1165^{*}$ \\
\hline $\mathrm{H} 221$ & 0.3878 & 0.6180 & 0.6634 & $0.0961^{*}$ \\
\hline $\mathrm{H} 231$ & 0.3613 & 0.4222 & 0.7119 & $0.1003^{*}$ \\
\hline $\mathrm{H} 241$ & 0.2398 & 0.3982 & 0.7972 & $0.0892^{*}$ \\
\hline $\mathrm{H} 251$ & 0.4621 & 0.9758 & 0.9098 & $0.0691 *$ \\
\hline $\mathrm{H} 252$ & 0.4751 & 0.8727 & 0.8509 & $0.0691 *$ \\
\hline $\mathrm{H} 271$ & 0.7674 & 0.8179 & 1.0567 & $0.0819^{*}$ \\
\hline $\mathrm{H} 272$ & 0.8637 & 0.8151 & 1.0007 & $0.0826^{*}$ \\
\hline H273 & 0.8236 & 0.6850 & 1.0351 & $0.0821 *$ \\
\hline H1011 & -0.2671 & 0.3219 & 0.6144 & $0.0981 *$ \\
\hline H1012 & -0.1117 & 0.2928 & 0.6452 & $0.0979 *$ \\
\hline H1013 & -0.2008 & 0.1881 & 0.5992 & $0.0982 *$ \\
\hline H1041 & 0.1422 & 0.3215 & 0.5924 & $0.0478^{*}$ \\
\hline H1051 & 0.0575 & 0.4492 & 0.4538 & $0.0514^{*}$ \\
\hline H1061 & 0.1396 & 0.2149 & 0.4596 & $0.0428^{*}$ \\
\hline H1141 & 0.3410 & 0.1196 & 0.3155 & $0.0940^{*}$ \\
\hline H1142 & 0.2614 & 0.0916 & 0.3717 & $0.0936^{*}$ \\
\hline H1143 & 0.1889 & 0.1311 & 0.2915 & $0.0936^{*}$ \\
\hline H1151 & 0.3760 & 0.3612 & 0.2829 & $0.1135^{*}$ \\
\hline H1152 & 0.2926 & 0.4627 & 0.3115 & $0.1137^{*}$ \\
\hline H1153 & 0.2242 & 0.3646 & 0.2522 & $0.1139^{*}$ \\
\hline H1161 & 0.4930 & 0.2011 & 0.5413 & $0.0455^{*}$ \\
\hline H1162 & 0.3764 & 0.1091 & 0.5006 & $0.0447^{*}$ \\
\hline H1181 & 0.4899 & 0.1526 & 0.6716 & $0.0480^{*}$ \\
\hline H1182 & 0.4170 & 0.0356 & 0.6273 & $0.0484 *$ \\
\hline H1201 & 0.4422 & 0.1657 & 0.7873 & $0.0534 *$ \\
\hline H1211 & 0.3084 & 0.1499 & 0.8666 & $0.0663 *$ \\
\hline H1221 & 0.0969 & 0.0750 & 0.8277 & $0.0864 *$ \\
\hline H1231 & 0.0120 & 0.0326 & 0.7035 & $0.0778^{*}$ \\
\hline
\end{tabular}




\begin{tabular}{|c|c|c|c|c|}
\hline H1241 & 0.1458 & 0.0568 & 0.6234 & $0.0614 *$ \\
\hline H1251 & 0.0319 & 0.4686 & 0.6389 & $0.0615 *$ \\
\hline H1252 & 0.0532 & 0.5618 & 0.5746 & $0.0617 *$ \\
\hline H1271 & -0.3419 & 0.3661 & 0.4805 & $0.0712^{*}$ \\
\hline H1272 & -0.2812 & 0.2311 & 0.4641 & $0.0709 *$ \\
\hline H1273 & -0.2314 & 0.3561 & 0.4323 & $0.0713^{*}$ \\
\hline
\end{tabular}

Atomic displacement parameters $\left(\AA^{2}\right)$

\begin{tabular}{|c|c|c|c|c|c|c|}
\hline & $U^{11}$ & $U^{22}$ & $U^{33}$ & $U^{12}$ & $U^{13}$ & $U^{23}$ \\
\hline $\mathrm{C} 1$ & $0.078(2)$ & 0.0607 (17) & $0.0530(16)$ & $0.0116(15)$ & $0.0308(15)$ & 0.0019 (13) \\
\hline $\mathrm{C} 2$ & $0.0313(10)$ & $0.0382(10)$ & $0.0431(11)$ & $0.0027(8)$ & $0.0194(9)$ & $0.0076(9)$ \\
\hline $\mathrm{O} 3$ & $0.0283(7)$ & $0.0490(9)$ & $0.0559(10)$ & 0.0048 (6) & $0.0189(7)$ & $0.0216(8)$ \\
\hline $\mathrm{C} 4$ & $0.0284(9)$ & $0.0488(12)$ & $0.0433(11)$ & $0.0027(9)$ & $0.0136(9)$ & $0.0113(10)$ \\
\hline $\mathrm{C} 5$ & $0.0257(9)$ & $0.0366(10)$ & $0.0410(11)$ & $-0.0003(7)$ & $0.0093(8)$ & $0.0066(8)$ \\
\hline C6 & $0.0271(9)$ & $0.0338(9)$ & $0.0334(10)$ & $0.0020(7)$ & $0.0102(8)$ & $0.0052(8)$ \\
\hline $\mathrm{C} 7$ & $0.0254(8)$ & $0.0364(9)$ & $0.0339(10)$ & $0.0029(7)$ & $0.0114(7)$ & $0.0049(8)$ \\
\hline $\mathrm{C} 8$ & $0.0312(10)$ & $0.0398(10)$ & $0.0368(11)$ & $0.0073(8)$ & $0.0131(8)$ & $0.0074(8)$ \\
\hline O9 & $0.0354(8)$ & $0.0352(7)$ & $0.0514(9)$ & $0.0055(6)$ & $0.0163(7)$ & $0.0139(7)$ \\
\hline $\mathrm{O} 10$ & $0.0369(9)$ & $0.0685(12)$ & $0.0545(10)$ & $0.0182(8)$ & $0.0147(8)$ & $0.0266(9)$ \\
\hline O11 & $0.0327(7)$ & $0.0530(9)$ & $0.0346(7)$ & $0.0110(7)$ & $0.0147(6)$ & $0.0101(7)$ \\
\hline $\mathrm{C} 12$ & $0.0371(10)$ & $0.0473(12)$ & $0.0330(10)$ & $0.0087(9)$ & $0.0124(8)$ & $0.0100(9)$ \\
\hline $\mathrm{O} 13$ & $0.0347(7)$ & $0.0498(9)$ & $0.0324(7)$ & $-0.0016(7)$ & $0.0082(6)$ & $0.0048(7)$ \\
\hline $\mathrm{C} 14$ & $0.0446(13)$ & $0.0526(14)$ & $0.0568(15)$ & $0.0087(11)$ & $0.0204(12)$ & $0.0253(12)$ \\
\hline $\mathrm{C} 15$ & $0.080(2)$ & 0.0647 (17) & $0.0388(13)$ & $0.0124(15)$ & $0.0235(13)$ & $0.0008(12)$ \\
\hline C16 & $0.0331(11)$ & 0.0443 (12) & $0.0484(13)$ & $-0.0063(9)$ & $0.0156(10)$ & $-0.0027(10)$ \\
\hline O17 & $0.0386(8)$ & $0.0621(11)$ & $0.0432(9)$ & $-0.0114(8)$ & $0.0124(7)$ & $-0.0155(8)$ \\
\hline $\mathrm{C} 18$ & $0.0537(14)$ & $0.0534(14)$ & $0.0440(13)$ & $-0.0082(12)$ & $0.0000(11)$ & $-0.0052(11)$ \\
\hline C19 & 0.0648 (16) & $0.0427(12)$ & $0.0341(12)$ & $0.0026(11)$ & $-0.0003(11)$ & $-0.0018(9)$ \\
\hline $\mathrm{C} 20$ & $0.128(3)$ & $0.0489(15)$ & $0.0520(17)$ & $0.0237(18)$ & 0.0317 (19) & 0.0098 (13) \\
\hline $\mathrm{C} 21$ & $0.148(4)$ & $0.061(2)$ & 0.0553 (19) & $0.007(2)$ & $0.039(2)$ & $0.0144(16)$ \\
\hline $\mathrm{C} 22$ & $0.097(3)$ & $0.076(2)$ & $0.0456(16)$ & 0.0005 (19) & $0.0204(16)$ & $-0.0011(15)$ \\
\hline $\mathrm{C} 23$ & $0.089(2)$ & 0.0637 (19) & $0.068(2)$ & $0.0196(18)$ & $0.0212(18)$ & $-0.0010(16)$ \\
\hline $\mathrm{C} 24$ & $0.084(2)$ & $0.0454(14)$ & 0.0615 (18) & 0.0088 (14) & $0.0117(16)$ & $0.0011(13)$ \\
\hline $\mathrm{C} 25$ & $0.0395(12)$ & $0.0686(17)$ & $0.0599(16)$ & $0.0118(12)$ & $0.0230(12)$ & $0.0316(14)$ \\
\hline $\mathrm{O} 26$ & $0.0361(8)$ & $0.0436(9)$ & $0.0670(12)$ & $0.0043(7)$ & $0.0249(8)$ & $0.0207(8)$ \\
\hline $\mathrm{C} 27$ & $0.0363(11)$ & $0.0541(14)$ & $0.0593(15)$ & $0.0000(11)$ & $0.0094(11)$ & $0.0084(12)$ \\
\hline C101 & $0.0612(16)$ & $0.0611(15)$ & $0.0474(14)$ & $0.0054(13)$ & 0.0267 (12) & $0.0070(12)$ \\
\hline $\mathrm{C} 102$ & $0.0303(10)$ & $0.0327(9)$ & 0.0409 (11) & $0.0050(7)$ & $0.0139(8)$ & $-0.0020(8)$ \\
\hline O103 & $0.0314(7)$ & $0.0345(7)$ & $0.0682(11)$ & $0.0012(6)$ & $0.0221(7)$ & $-0.0129(8)$ \\
\hline C104 & $0.0286(9)$ & $0.0349(10)$ & $0.0476(12)$ & $0.0033(8)$ & $0.0123(9)$ & $-0.0044(9)$ \\
\hline $\mathrm{C} 105$ & $0.0321(10)$ & $0.0272(9)$ & $0.0524(13)$ & $0.0042(8)$ & $0.0107(9)$ & $0.0002(9)$ \\
\hline $\mathrm{C} 106$ & $0.0284(9)$ & $0.0278(8)$ & $0.0348(10)$ & $0.0006(7)$ & $0.0076(8)$ & $0.0029(7)$ \\
\hline C107 & $0.0284(9)$ & $0.0292(9)$ & $0.034(1)$ & $-0.0004(7)$ & $0.0133(8)$ & $0.0033(7)$ \\
\hline C108 & $0.0369(11)$ & $0.0317(9)$ & $0.0430(11)$ & $-0.0067(8)$ & $0.0165(9)$ & $0.0016(8)$ \\
\hline O109 & $0.0423(9)$ & $0.0258(7)$ & $0.0646(11)$ & $-0.0037(6)$ & $0.0180(8)$ & $-0.0051(7)$ \\
\hline O110 & $0.0431(9)$ & $0.0531(10)$ & $0.0629(12)$ & $-0.0206(8)$ & $0.0187(8)$ & $-0.0122(9)$ \\
\hline O111 & $0.0383(8)$ & $0.0483(9)$ & $0.0354(8)$ & $-0.0037(7)$ & $0.0177(7)$ & $0.0062(6)$ \\
\hline
\end{tabular}


supporting information

\begin{tabular}{lllllll}
\hline & & & & \\
$\mathrm{C} 112$ & $0.0479(12)$ & $0.0412(11)$ & $0.0338(10)$ & $-0.0025(10)$ & $0.0130(9)$ & $0.0056(9)$ \\
$\mathrm{O} 113$ & $0.0428(9)$ & $0.0503(9)$ & $0.0357(8)$ & $0.0075(7)$ & $0.0049(7)$ & $0.0058(7)$ \\
C114 & $0.0631(16)$ & $0.0447(13)$ & $0.0480(14)$ & $-0.0056(11)$ & $0.0220(12)$ & $-0.0037(11)$ \\
C115 & $0.092(2)$ & $0.0594(17)$ & $0.0422(14)$ & $-0.0056(16)$ & $0.0214(14)$ & $0.0162(12)$ \\
C116 & $0.0292(9)$ & $0.0361(9)$ & $0.0354(10)$ & $0.0062(7)$ & $0.0141(8)$ & $0.0056(8)$ \\
O117 & $0.0380(8)$ & $0.0414(8)$ & $0.0346(7)$ & $0.0132(6)$ & $0.0163(6)$ & $0.0118(6)$ \\
C118 & $0.0330(10)$ & $0.0402(10)$ & $0.0331(10)$ & $0.0080(8)$ & $0.0056(8)$ & $0.0072(8)$ \\
C119 & $0.0369(10)$ & $0.0304(9)$ & $0.0338(10)$ & $0.0053(8)$ & $0.0091(8)$ & $0.0066(8)$ \\
C120 & $0.0389(11)$ & $0.0478(12)$ & $0.0345(11)$ & $0.0105(9)$ & $0.0038(9)$ & $0.0045(9)$ \\
C121 & $0.0577(15)$ & $0.0671(16)$ & $0.0345(11)$ & $0.0239(13)$ & $0.0142(11)$ & $0.0094(11)$ \\
C122 & $0.0568(16)$ & $0.0704(18)$ & $0.0559(16)$ & $0.0205(14)$ & $0.0294(14)$ & $0.0281(14)$ \\
C123 & $0.0424(13)$ & $0.0590(16)$ & $0.0701(19)$ & $-0.0047(12)$ & $0.0200(13)$ & $0.0119(14)$ \\
C124 & $0.0415(12)$ & $0.0479(12)$ & $0.0411(12)$ & $-0.0063(10)$ & $0.0097(10)$ & $0.0014(10)$ \\
C125 & $0.0308(10)$ & $0.0470(12)$ & $0.0586(14)$ & $0.0041(9)$ & $0.0081(10)$ & $-0.0192(11)$ \\
O126 & $0.0301(7)$ & $0.0334(7)$ & $0.0569(10)$ & $0.0032(6)$ & $0.0122(7)$ & $-0.0111(7)$ \\
C127 & $0.0435(12)$ & $0.0371(11)$ & $0.0496(13)$ & $-0.0023(9)$ & $0.0036(10)$ & $0.0009(10)$ \\
& & & & & \\
\hline
\end{tabular}

Geometric parameters $\left(\AA,{ }^{\circ}\right)$

\begin{tabular}{llll}
\hline $\mathrm{C} 1-\mathrm{C} 2$ & $1.515(4)$ & $\mathrm{C} 101-\mathrm{C} 102$ & $1.509(3)$ \\
$\mathrm{C} 1-\mathrm{H} 11$ & 1.018 & $\mathrm{C} 101-\mathrm{H} 1011$ & 0.999 \\
$\mathrm{C} 1-\mathrm{H} 12$ & 0.978 & $\mathrm{C} 101-\mathrm{H} 1012$ & 0.957 \\
$\mathrm{C} 1-\mathrm{H} 13$ & 0.932 & $\mathrm{C} 101-\mathrm{H} 1013$ & 0.975 \\
$\mathrm{C} 2-\mathrm{O} 3$ & $1.432(2)$ & $\mathrm{C} 102-\mathrm{O} 103$ & $1.435(2)$ \\
$\mathrm{C} 2-\mathrm{O} 26$ & $1.417(3)$ & $\mathrm{C} 102-\mathrm{O} 126$ & $1.427(3)$ \\
$\mathrm{C} 2-\mathrm{C} 27$ & $1.498(3)$ & $\mathrm{C} 102-\mathrm{C} 127$ & $1.496(3)$ \\
$\mathrm{O} 3-\mathrm{C} 4$ & $1.428(3)$ & $\mathrm{O} 103-\mathrm{C} 104$ & $1.428(3)$ \\
$\mathrm{C} 4-\mathrm{C} 5$ & $1.509(3)$ & $\mathrm{C} 104-\mathrm{C} 105$ & $1.522(3)$ \\
$\mathrm{C} 4-\mathrm{C} 25$ & $1.540(3)$ & $\mathrm{C} 104-\mathrm{C} 125$ & $1.534(3)$ \\
$\mathrm{C} 4-\mathrm{H} 41$ & 0.987 & $\mathrm{C} 104-\mathrm{H} 1041$ & 1.003 \\
$\mathrm{C} 5-\mathrm{C} 6$ & $1.527(3)$ & $\mathrm{C} 105-\mathrm{C} 106$ & $1.518(3)$ \\
$\mathrm{C} 5-\mathrm{O} 9$ & $1.456(3)$ & $\mathrm{C} 105-\mathrm{O} 109$ & $1.461(3)$ \\
$\mathrm{C} 5-\mathrm{H} 51$ & 1.008 & $\mathrm{C} 105-\mathrm{H} 1051$ & 0.998 \\
$\mathrm{C} 6-\mathrm{C} 7$ & $1.533(3)$ & $\mathrm{C} 106-\mathrm{C} 107$ & $1.537(3)$ \\
$\mathrm{C} 6-\mathrm{O} 13$ & $1.429(2)$ & $\mathrm{C} 106-\mathrm{O} 113$ & $1.429(2)$ \\
$\mathrm{C} 6-\mathrm{H} 61$ & 0.995 & $\mathrm{C} 106-\mathrm{H} 1061$ & 0.985 \\
$\mathrm{C} 7-\mathrm{C} 8$ & $1.535(3)$ & $\mathrm{C} 107-\mathrm{C} 108$ & $1.532(3)$ \\
$\mathrm{C} 7-\mathrm{O} 11$ & $1.421(2)$ & $\mathrm{C} 107-\mathrm{O} 111$ & $1.420(2)$ \\
$\mathrm{C} 7-\mathrm{C} 16$ & $1.511(3)$ & $\mathrm{C} 107-\mathrm{C} 116$ & $1.506(3)$ \\
$\mathrm{C} 8-\mathrm{O} 9$ & $1.349(2)$ & $\mathrm{C} 108-\mathrm{O} 109$ & $1.347(3)$ \\
$\mathrm{C} 8-\mathrm{O} 10$ & $1.197(3)$ & $\mathrm{C} 108-\mathrm{O} 110$ & $1.196(3)$ \\
$\mathrm{O} 11-\mathrm{C} 12$ & $1.441(3)$ & $\mathrm{O} 111-\mathrm{C} 112$ & $1.439(3)$ \\
$\mathrm{C} 12-\mathrm{O} 13$ & $1.422(2)$ & $\mathrm{C} 112-\mathrm{O} 113$ & $1.419(3)$ \\
$\mathrm{C} 12-\mathrm{C} 14$ & $1.518(3)$ & $\mathrm{C} 112-\mathrm{C} 114$ & $1.518(3)$ \\
$\mathrm{C} 12-\mathrm{C} 15$ & $1.503(4)$ & $\mathrm{C} 112-\mathrm{C} 115$ & 0.974 \\
$\mathrm{C} 14-\mathrm{H} 141$ & 0.976 & $\mathrm{C} 114-\mathrm{H} 1141$ & 0.959 \\
$\mathrm{C} 14-\mathrm{H} 142$ & 0.967 & $\mathrm{C} 114-\mathrm{H} 1142$ & \\
$\mathrm{C} 14-\mathrm{H} 143$ & 0.956 & &
\end{tabular}




\begin{tabular}{|c|c|c|c|}
\hline $\mathrm{C} 15-\mathrm{H} 151$ & 0.948 & $\mathrm{C} 115-\mathrm{H} 1151$ & 0.934 \\
\hline $\mathrm{C} 15-\mathrm{H} 152$ & 0.933 & $\mathrm{C} 115-\mathrm{H} 1152$ & 0.986 \\
\hline $\mathrm{C} 15-\mathrm{H} 153$ & 0.974 & $\mathrm{C} 115-\mathrm{H} 1153$ & 0.939 \\
\hline $\mathrm{C} 16-\mathrm{O} 17$ & $1.414(3)$ & $\mathrm{C} 116-\mathrm{O} 117$ & $1.418(2)$ \\
\hline $\mathrm{C} 16-\mathrm{H} 161$ & 0.992 & $\mathrm{C} 116-\mathrm{H} 1161$ & 0.991 \\
\hline $\mathrm{C} 16-\mathrm{H} 162$ & 1.016 & $\mathrm{C} 116-\mathrm{H} 1162$ & 1.010 \\
\hline $\mathrm{O} 17-\mathrm{C} 18$ & $1.420(3)$ & $\mathrm{O} 117-\mathrm{C} 118$ & $1.419(2)$ \\
\hline $\mathrm{C} 18-\mathrm{C} 19$ & $1.496(4)$ & $\mathrm{C} 118-\mathrm{C} 119$ & $1.501(3)$ \\
\hline $\mathrm{C} 18-\mathrm{H} 181$ & 1.025 & $\mathrm{C} 118-\mathrm{H} 1181$ & 1.001 \\
\hline $\mathrm{C} 18-\mathrm{H} 182$ & 0.972 & $\mathrm{C} 118-\mathrm{H} 1182$ & 0.989 \\
\hline $\mathrm{C} 19-\mathrm{C} 20$ & $1.383(4)$ & $\mathrm{C} 119-\mathrm{C} 120$ & $1.377(3)$ \\
\hline $\mathrm{C} 19-\mathrm{C} 24$ & $1.374(4)$ & $\mathrm{C} 119-\mathrm{C} 124$ & $1.392(3)$ \\
\hline $\mathrm{C} 20-\mathrm{C} 21$ & $1.384(5)$ & $\mathrm{C} 120-\mathrm{C} 121$ & $1.389(3)$ \\
\hline $\mathrm{C} 20-\mathrm{H} 201$ & 0.917 & $\mathrm{C} 120-\mathrm{H} 1201$ & 0.902 \\
\hline $\mathrm{C} 21-\mathrm{C} 22$ & $1.378(5)$ & $\mathrm{C} 121-\mathrm{C} 122$ & $1.371(4)$ \\
\hline $\mathrm{C} 21-\mathrm{H} 211$ & 0.941 & C121-H1211 & 0.934 \\
\hline $\mathrm{C} 22-\mathrm{C} 23$ & $1.354(5)$ & $\mathrm{C} 122-\mathrm{C} 123$ & $1.381(4)$ \\
\hline $\mathrm{C} 22-\mathrm{H} 221$ & 0.930 & $\mathrm{C} 122-\mathrm{H} 1221$ & 0.934 \\
\hline $\mathrm{C} 23-\mathrm{C} 24$ & $1.386(5)$ & $\mathrm{C} 123-\mathrm{C} 124$ & $1.395(3)$ \\
\hline $\mathrm{C} 23-\mathrm{H} 231$ & 0.927 & C123-H1231 & 0.929 \\
\hline $\mathrm{C} 24-\mathrm{H} 241$ & 0.955 & $\mathrm{C} 124-\mathrm{H} 1241$ & 0.937 \\
\hline $\mathrm{C} 25-\mathrm{O} 26$ & $1.403(3)$ & $\mathrm{C} 125-\mathrm{O} 126$ & $1.431(3)$ \\
\hline $\mathrm{C} 25-\mathrm{H} 251$ & 0.973 & $\mathrm{C} 125-\mathrm{H} 1251$ & 0.996 \\
\hline $\mathrm{C} 25-\mathrm{H} 252$ & 0.989 & $\mathrm{C} 125-\mathrm{H} 1252$ & 1.008 \\
\hline $\mathrm{C} 27-\mathrm{H} 271$ & 0.976 & $\mathrm{C} 127-\mathrm{H} 1271$ & 0.984 \\
\hline $\mathrm{C} 27-\mathrm{H} 272$ & 0.978 & $\mathrm{C} 127-\mathrm{H} 1272$ & 0.957 \\
\hline $\mathrm{C} 27-\mathrm{H} 273$ & 0.983 & $\mathrm{C} 127-\mathrm{H} 1273$ & 0.983 \\
\hline $\mathrm{C} 2-\mathrm{C} 1-\mathrm{H} 11$ & 111.4 & $\mathrm{C} 102-\mathrm{C} 101-\mathrm{H} 1011$ & 109.8 \\
\hline $\mathrm{C} 2-\mathrm{C} 1-\mathrm{H} 12$ & 111.8 & $\mathrm{C} 102-\mathrm{C} 101-\mathrm{H} 1012$ & 108.0 \\
\hline $\mathrm{H} 11-\mathrm{C} 1-\mathrm{H} 12$ & 105.1 & H1011-C101-H1012 & 110.8 \\
\hline $\mathrm{C} 2-\mathrm{C} 1-\mathrm{H} 13$ & 111.3 & C102-C101-H1013 & 110.7 \\
\hline $\mathrm{H} 11-\mathrm{C} 1-\mathrm{H} 13$ & 108.6 & $\mathrm{H} 1011-\mathrm{C} 101-\mathrm{H} 1013$ & 109.8 \\
\hline $\mathrm{H} 12-\mathrm{C} 1-\mathrm{H} 13$ & 108.4 & $\mathrm{H} 1012-\mathrm{C} 101-\mathrm{H} 1013$ & 107.7 \\
\hline $\mathrm{C} 1-\mathrm{C} 2-\mathrm{O} 3$ & $109.6(2)$ & $\mathrm{C} 101-\mathrm{C} 102-\mathrm{O} 103$ & $109.69(19)$ \\
\hline $\mathrm{C} 1-\mathrm{C} 2-\mathrm{O} 26$ & $111.2(2)$ & $\mathrm{C} 101-\mathrm{C} 102-\mathrm{O} 126$ & $111.6(2)$ \\
\hline $\mathrm{O} 3-\mathrm{C} 2-\mathrm{O} 26$ & $104.72(16)$ & $\mathrm{O} 103-\mathrm{C} 102-\mathrm{O} 126$ & $104.83(16)$ \\
\hline $\mathrm{C} 1-\mathrm{C} 2-\mathrm{C} 27$ & $113.0(2)$ & $\mathrm{C} 101-\mathrm{C} 102-\mathrm{C} 127$ & $112.2(2)$ \\
\hline $\mathrm{O} 3-\mathrm{C} 2-\mathrm{C} 27$ & $109.27(19)$ & $\mathrm{O} 103-\mathrm{C} 102-\mathrm{C} 127$ & $109.69(18)$ \\
\hline $\mathrm{O} 26-\mathrm{C} 2-\mathrm{C} 27$ & $108.7(2)$ & $\mathrm{O} 126-\mathrm{C} 102-\mathrm{C} 127$ & $108.53(18)$ \\
\hline $\mathrm{C} 2-\mathrm{O} 3-\mathrm{C} 4$ & $108.29(16)$ & $\mathrm{C} 102-\mathrm{O} 103-\mathrm{C} 104$ & $109.05(15)$ \\
\hline $\mathrm{O} 3-\mathrm{C} 4-\mathrm{C} 5$ & $107.26(19)$ & $\mathrm{O} 103-\mathrm{C} 104-\mathrm{C} 105$ & $107.69(19)$ \\
\hline $\mathrm{O} 3-\mathrm{C} 4-\mathrm{C} 25$ & $104.32(16)$ & $\mathrm{O} 103-\mathrm{C} 104-\mathrm{C} 125$ & $104.46(16)$ \\
\hline $\mathrm{C} 5-\mathrm{C} 4-\mathrm{C} 25$ & $112.6(2)$ & $\mathrm{C} 105-\mathrm{C} 104-\mathrm{C} 125$ & $112.05(19)$ \\
\hline $\mathrm{O} 3-\mathrm{C} 4-\mathrm{H} 41$ & 110.4 & $\mathrm{O} 103-\mathrm{C} 104-\mathrm{H} 1041$ & 109.3 \\
\hline $\mathrm{C} 5-\mathrm{C} 4-\mathrm{H} 41$ & 110.7 & $\mathrm{C} 105-\mathrm{C} 104-\mathrm{H} 1041$ & 112.0 \\
\hline $\mathrm{C} 25-\mathrm{C} 4-\mathrm{H} 41$ & 111.2 & $\mathrm{C} 125-\mathrm{C} 104-\mathrm{H} 1041$ & 111.0 \\
\hline $\mathrm{C} 4-\mathrm{C} 5-\mathrm{C} 6$ & $114.72(18)$ & $\mathrm{C} 104-\mathrm{C} 105-\mathrm{C} 106$ & $115.28(17)$ \\
\hline
\end{tabular}




\begin{tabular}{|c|c|}
\hline $\mathrm{C} 4-\mathrm{C} 5-\mathrm{O} 9$ & $107.97(18)$ \\
\hline $\mathrm{C} 6-\mathrm{C} 5-\mathrm{O} 9$ & $106.00(15)$ \\
\hline $\mathrm{C} 4-\mathrm{C} 5-\mathrm{H} 51$ & 106.3 \\
\hline $\mathrm{C} 6-\mathrm{C} 5-\mathrm{H} 51$ & 111.3 \\
\hline $\mathrm{O} 9-\mathrm{C} 5-\mathrm{H} 51$ & 110.5 \\
\hline $\mathrm{C} 5-\mathrm{C} 6-\mathrm{C} 7$ & $105.60(16)$ \\
\hline $\mathrm{C} 5-\mathrm{C} 6-\mathrm{O} 13$ & $109.23(17)$ \\
\hline $\mathrm{C} 7-\mathrm{C} 6-\mathrm{O} 13$ & $103.30(15)$ \\
\hline $\mathrm{C} 5-\mathrm{C} 6-\mathrm{H} 61$ & 111.7 \\
\hline C7- 6 6- 661 & 112.1 \\
\hline $\mathrm{O} 13-\mathrm{C} 6-\mathrm{H} 61$ & 114.2 \\
\hline $\mathrm{C} 6-\mathrm{C} 7-\mathrm{C} 8$ & $103.62(16)$ \\
\hline $\mathrm{C} 6-\mathrm{C} 7-\mathrm{O} 11$ & $105.91(16)$ \\
\hline $\mathrm{C} 8-\mathrm{C} 7-\mathrm{O} 11$ & $108.09(16)$ \\
\hline $\mathrm{C} 6-\mathrm{C} 7-\mathrm{C} 16$ & $116.74(17)$ \\
\hline $\mathrm{C} 8-\mathrm{C} 7-\mathrm{C} 16$ & $109.94(18)$ \\
\hline $\mathrm{O} 11-\mathrm{C} 7-\mathrm{C} 16$ & $111.94(16)$ \\
\hline $\mathrm{C} 7-\mathrm{C} 8-\mathrm{O} 9$ & $110.60(17)$ \\
\hline $\mathrm{C} 7-\mathrm{C} 8-\mathrm{O} 10$ & $127.0(2)$ \\
\hline $\mathrm{O} 9-\mathrm{C} 8-\mathrm{O} 10$ & $122.4(2)$ \\
\hline $\mathrm{C} 5-\mathrm{O} 9-\mathrm{C} 8$ & $111.86(16)$ \\
\hline $\mathrm{C} 7-\mathrm{O} 11-\mathrm{C} 12$ & $107.78(15)$ \\
\hline $\mathrm{O} 11-\mathrm{C} 12-\mathrm{O} 13$ & $104.60(16)$ \\
\hline $\mathrm{O} 11-\mathrm{C} 12-\mathrm{C} 14$ & $109.6(2)$ \\
\hline $\mathrm{O} 13-\mathrm{C} 12-\mathrm{C} 14$ & $111.07(18)$ \\
\hline $\mathrm{O} 11-\mathrm{C} 12-\mathrm{C} 15$ & $109.02(19)$ \\
\hline $\mathrm{O} 13-\mathrm{C} 12-\mathrm{C} 15$ & $108.7(2)$ \\
\hline $\mathrm{C} 14-\mathrm{C} 12-\mathrm{C} 15$ & $113.4(2)$ \\
\hline $\mathrm{C} 6-\mathrm{O} 13-\mathrm{C} 12$ & $107.35(16)$ \\
\hline $\mathrm{C} 12-\mathrm{C} 14-\mathrm{H} 141$ & 113.3 \\
\hline $\mathrm{C} 12-\mathrm{C} 14-\mathrm{H} 142$ & 108.2 \\
\hline $\mathrm{H} 141-\mathrm{C} 14-\mathrm{H} 142$ & 106.5 \\
\hline $\mathrm{C} 12-\mathrm{C} 14-\mathrm{H} 143$ & 111.4 \\
\hline $\mathrm{H} 141-\mathrm{C} 14-\mathrm{H} 143$ & 109.8 \\
\hline $\mathrm{H} 142-\mathrm{C} 14-\mathrm{H} 143$ & 107.3 \\
\hline $\mathrm{C} 12-\mathrm{C} 15-\mathrm{H} 151$ & 110.8 \\
\hline $\mathrm{C} 12-\mathrm{C} 15-\mathrm{H} 152$ & 109.5 \\
\hline $\mathrm{H} 151-\mathrm{C} 15-\mathrm{H} 152$ & 109.7 \\
\hline $\mathrm{C} 12-\mathrm{C} 15-\mathrm{H} 153$ & 108.8 \\
\hline $\mathrm{H} 151-\mathrm{C} 15-\mathrm{H} 153$ & 109.0 \\
\hline $\mathrm{H} 152-\mathrm{C} 15-\mathrm{H} 153$ & 109.0 \\
\hline $\mathrm{C} 7-\mathrm{C} 16-\mathrm{O} 17$ & $104.76(16)$ \\
\hline $\mathrm{C} 7-\mathrm{C} 16-\mathrm{H} 161$ & 108.3 \\
\hline $\mathrm{O} 17-\mathrm{C} 16-\mathrm{H} 161$ & 115.5 \\
\hline C7-C16-H162 & 110.0 \\
\hline $\mathrm{O} 17-\mathrm{C} 16-\mathrm{H} 162$ & 112.3 \\
\hline $\mathrm{H} 161-\mathrm{C} 16-\mathrm{H} 162$ & 105.9 \\
\hline $\mathrm{C} 16-\mathrm{O} 17-\mathrm{C} 18$ & $115.72(18)$ \\
\hline
\end{tabular}

\begin{tabular}{|c|c|}
\hline $\mathrm{C} 104-\mathrm{C} 105-\mathrm{O} 109$ & $107.44(19)$ \\
\hline $\mathrm{C} 106-\mathrm{C} 105-\mathrm{O} 109$ & $105.91(16)$ \\
\hline $\mathrm{C} 104-\mathrm{C} 105-\mathrm{H} 1051$ & 108.2 \\
\hline $\mathrm{C} 106-\mathrm{C} 105-\mathrm{H} 1051$ & 107.5 \\
\hline $\mathrm{O} 109-\mathrm{C} 105-\mathrm{H} 1051$ & 112.6 \\
\hline $\mathrm{C} 105-\mathrm{C} 106-\mathrm{C} 107$ & $105.63(16)$ \\
\hline $\mathrm{C} 105-\mathrm{C} 106-\mathrm{O} 113$ & $108.98(17)$ \\
\hline $\mathrm{C} 107-\mathrm{C} 106-\mathrm{O} 113$ & $103.04(15)$ \\
\hline $\mathrm{C} 105-\mathrm{C} 106-\mathrm{H} 1061$ & 110.9 \\
\hline $\mathrm{C} 107-\mathrm{C} 106-\mathrm{H} 1061$ & 114.4 \\
\hline $\mathrm{O} 113-\mathrm{C} 106-\mathrm{H} 1061$ & 113.4 \\
\hline $\mathrm{C} 106-\mathrm{C} 107-\mathrm{C} 108$ & $103.30(16)$ \\
\hline $\mathrm{C} 106-\mathrm{C} 107-\mathrm{O} 111$ & $106.07(16)$ \\
\hline $\mathrm{C} 108-\mathrm{C} 107-\mathrm{O} 111$ & $108.78(15)$ \\
\hline $\mathrm{C} 106-\mathrm{C} 107-\mathrm{C} 116$ & $116.72(15)$ \\
\hline $\mathrm{C} 108-\mathrm{C} 107-\mathrm{C} 116$ & $110.32(18)$ \\
\hline $\mathrm{O} 111-\mathrm{C} 107-\mathrm{C} 116$ & $111.13(15)$ \\
\hline $\mathrm{C} 107-\mathrm{C} 108-\mathrm{O} 109$ & $110.89(17)$ \\
\hline $\mathrm{C} 107-\mathrm{C} 108-\mathrm{O} 110$ & $127.3(2)$ \\
\hline $\mathrm{O} 109-\mathrm{C} 108-\mathrm{O} 110$ & $121.8(2)$ \\
\hline $\mathrm{C} 105-\mathrm{O} 109-\mathrm{C} 108$ & $111.30(16)$ \\
\hline $\mathrm{C} 107-\mathrm{O} 111-\mathrm{C} 112$ & $107.57(15)$ \\
\hline $\mathrm{O} 111-\mathrm{C} 112-\mathrm{O} 113$ & $105.24(17)$ \\
\hline $\mathrm{O} 111-\mathrm{C} 112-\mathrm{C} 114$ & $110.5(2)$ \\
\hline $\mathrm{O} 113-\mathrm{C} 112-\mathrm{C} 114$ & $111.4(2)$ \\
\hline $\mathrm{O} 111-\mathrm{C} 112-\mathrm{C} 115$ & $108.2(2)$ \\
\hline $\mathrm{O} 113-\mathrm{C} 112-\mathrm{C} 115$ & $108.3(2)$ \\
\hline $\mathrm{C} 114-\mathrm{C} 112-\mathrm{C} 115$ & $112.8(2)$ \\
\hline $\mathrm{C} 106-\mathrm{O} 113-\mathrm{C} 112$ & $107.15(16)$ \\
\hline $\mathrm{C} 112-\mathrm{C} 114-\mathrm{H} 1141$ & 111.0 \\
\hline $\mathrm{C} 112-\mathrm{C} 114-\mathrm{H} 1142$ & 112.2 \\
\hline $\mathrm{H} 1141-\mathrm{C} 114-\mathrm{H} 1142$ & 104.5 \\
\hline C112-C114-H1143 & 112.2 \\
\hline H1141-C114-H1143 & 105.3 \\
\hline $\mathrm{H} 1142-\mathrm{C} 114-\mathrm{H} 1143$ & 111.2 \\
\hline $\mathrm{C} 112-\mathrm{C} 115-\mathrm{H} 1151$ & 111.8 \\
\hline C112-C115-H1152 & 106.9 \\
\hline $\mathrm{H} 1151-\mathrm{C} 115-\mathrm{H} 1152$ & 107.4 \\
\hline $\mathrm{C} 112-\mathrm{C} 115-\mathrm{H} 1153$ & 110.7 \\
\hline $\mathrm{H} 1151-\mathrm{C} 115-\mathrm{H} 1153$ & 111.1 \\
\hline H1152-C115-H1153 & 108.7 \\
\hline C107-C116-O117 & $104.97(15)$ \\
\hline C107-C116-H1161 & 111.3 \\
\hline $\mathrm{O} 117-\mathrm{C} 116-\mathrm{H} 1161$ & 112.4 \\
\hline C107-C116-H1162 & 109.1 \\
\hline $\mathrm{O} 117-\mathrm{C} 116-\mathrm{H} 1162$ & 113.5 \\
\hline $\mathrm{H} 1161-\mathrm{C} 116-\mathrm{H} 1162$ & 105.7 \\
\hline $\mathrm{C} 116-\mathrm{O} 117-\mathrm{C} 118$ & $113.76(15)$ \\
\hline
\end{tabular}




\begin{tabular}{|c|c|c|c|}
\hline $\mathrm{O} 17-\mathrm{C} 18-\mathrm{C} 19$ & $107.1(2)$ & $\mathrm{O} 117-\mathrm{C} 118-\mathrm{C} 119$ & $108.54(16)$ \\
\hline $\mathrm{O} 17-\mathrm{C} 18-\mathrm{H} 181$ & 107.8 & $\mathrm{O} 117-\mathrm{C} 118-\mathrm{H} 1181$ & 111.5 \\
\hline $\mathrm{C} 19-\mathrm{C} 18-\mathrm{H} 181$ & 110.1 & $\mathrm{C} 119-\mathrm{C} 118-\mathrm{H} 1181$ & 111.0 \\
\hline $\mathrm{O} 17-\mathrm{C} 18-\mathrm{H} 182$ & 111.4 & $\mathrm{O} 117-\mathrm{C} 118-\mathrm{H} 1182$ & 109.3 \\
\hline $\mathrm{C} 19-\mathrm{C} 18-\mathrm{H} 182$ & 114.2 & $\mathrm{C} 119-\mathrm{C} 118-\mathrm{H} 1182$ & 112.1 \\
\hline $\mathrm{H} 181-\mathrm{C} 18-\mathrm{H} 182$ & 106.1 & $\mathrm{H} 1181-\mathrm{C} 118-\mathrm{H} 1182$ & 104.4 \\
\hline $\mathrm{C} 18-\mathrm{C} 19-\mathrm{C} 20$ & $120.2(3)$ & $\mathrm{C} 118-\mathrm{C} 119-\mathrm{C} 120$ & $121.2(2)$ \\
\hline $\mathrm{C} 18-\mathrm{C} 19-\mathrm{C} 24$ & $121.6(3)$ & $\mathrm{C} 118-\mathrm{C} 119-\mathrm{C} 124$ & $119.5(2)$ \\
\hline $\mathrm{C} 20-\mathrm{C} 19-\mathrm{C} 24$ & $118.2(3)$ & $\mathrm{C} 120-\mathrm{C} 119-\mathrm{C} 124$ & $119.3(2)$ \\
\hline $\mathrm{C} 19-\mathrm{C} 20-\mathrm{C} 21$ & $121.0(3)$ & $\mathrm{C} 119-\mathrm{C} 120-\mathrm{C} 121$ & $120.7(2)$ \\
\hline $\mathrm{C} 19-\mathrm{C} 20-\mathrm{H} 201$ & 118.9 & $\mathrm{C} 119-\mathrm{C} 120-\mathrm{H} 1201$ & 119.9 \\
\hline $\mathrm{C} 21-\mathrm{C} 20-\mathrm{H} 201$ & 120.1 & $\mathrm{C} 121-\mathrm{C} 120-\mathrm{H} 1201$ & 119.3 \\
\hline $\mathrm{C} 20-\mathrm{C} 21-\mathrm{C} 22$ & $119.8(3)$ & $\mathrm{C} 120-\mathrm{C} 121-\mathrm{C} 122$ & $120.2(3)$ \\
\hline $\mathrm{C} 20-\mathrm{C} 21-\mathrm{H} 211$ & 117.8 & $\mathrm{C} 120-\mathrm{C} 121-\mathrm{H} 1211$ & 119.2 \\
\hline $\mathrm{C} 22-\mathrm{C} 21-\mathrm{H} 211$ & 122.5 & $\mathrm{C} 122-\mathrm{C} 121-\mathrm{H} 1211$ & 120.5 \\
\hline $\mathrm{C} 21-\mathrm{C} 22-\mathrm{C} 23$ & $119.6(3)$ & $\mathrm{C} 121-\mathrm{C} 122-\mathrm{C} 123$ & $119.8(2)$ \\
\hline $\mathrm{C} 21-\mathrm{C} 22-\mathrm{H} 221$ & 119.9 & $\mathrm{C} 121-\mathrm{C} 122-\mathrm{H} 1221$ & 117.9 \\
\hline $\mathrm{C} 23-\mathrm{C} 22-\mathrm{H} 221$ & 120.4 & $\mathrm{C} 123-\mathrm{C} 122-\mathrm{H} 1221$ & 122.3 \\
\hline $\mathrm{C} 22-\mathrm{C} 23-\mathrm{C} 24$ & $120.7(3)$ & $\mathrm{C} 122-\mathrm{C} 123-\mathrm{C} 124$ & $120.4(3)$ \\
\hline $\mathrm{C} 22-\mathrm{C} 23-\mathrm{H} 231$ & 120.1 & $\mathrm{C} 122-\mathrm{C} 123-\mathrm{H} 1231$ & 120.9 \\
\hline $\mathrm{C} 24-\mathrm{C} 23-\mathrm{H} 231$ & 119.1 & $\mathrm{C} 124-\mathrm{C} 123-\mathrm{H} 1231$ & 118.7 \\
\hline $\mathrm{C} 23-\mathrm{C} 24-\mathrm{C} 19$ & $120.7(3)$ & $\mathrm{C} 123-\mathrm{C} 124-\mathrm{C} 119$ & $119.6(2)$ \\
\hline $\mathrm{C} 23-\mathrm{C} 24-\mathrm{H} 241$ & 118.6 & $\mathrm{C} 123-\mathrm{C} 124-\mathrm{H} 1241$ & 120.0 \\
\hline $\mathrm{C} 19-\mathrm{C} 24-\mathrm{H} 241$ & 120.7 & $\mathrm{C} 119-\mathrm{C} 124-\mathrm{H} 1241$ & 120.3 \\
\hline $\mathrm{C} 4-\mathrm{C} 25-\mathrm{O} 26$ & $103.96(18)$ & $\mathrm{C} 104-\mathrm{C} 125-\mathrm{O} 126$ & $102.55(18)$ \\
\hline $\mathrm{C} 4-\mathrm{C} 25-\mathrm{H} 251$ & 109.8 & $\mathrm{C} 104-\mathrm{C} 125-\mathrm{H} 1251$ & 108.3 \\
\hline $\mathrm{O} 26-\mathrm{C} 25-\mathrm{H} 251$ & 111.5 & $\mathrm{O} 126-\mathrm{C} 125-\mathrm{H} 1251$ & 115.9 \\
\hline $\mathrm{C} 4-\mathrm{C} 25-\mathrm{H} 252$ & 109.9 & $\mathrm{C} 104-\mathrm{C} 125-\mathrm{H} 1252$ & 110.4 \\
\hline $\mathrm{O} 26-\mathrm{C} 25-\mathrm{H} 252$ & 113.9 & $\mathrm{O} 126-\mathrm{C} 125-\mathrm{H} 1252$ & 111.6 \\
\hline $\mathrm{H} 251-\mathrm{C} 25-\mathrm{H} 252$ & 107.8 & $\mathrm{H} 1251-\mathrm{C} 125-\mathrm{H} 1252$ & 107.9 \\
\hline $\mathrm{C} 2-\mathrm{O} 26-\mathrm{C} 25$ & $107.06(18)$ & $\mathrm{C} 125-\mathrm{O} 126-\mathrm{C} 102$ & $106.07(17)$ \\
\hline $\mathrm{C} 2-\mathrm{C} 27-\mathrm{H} 271$ & 105.8 & $\mathrm{C} 102-\mathrm{C} 127-\mathrm{H} 1271$ & 107.8 \\
\hline $\mathrm{C} 2-\mathrm{C} 27-\mathrm{H} 272$ & 107.6 & $\mathrm{C} 102-\mathrm{C} 127-\mathrm{H} 1272$ & 109.6 \\
\hline $\mathrm{H} 271-\mathrm{C} 27-\mathrm{H} 272$ & 112.5 & $\mathrm{H} 1271-\mathrm{C} 127-\mathrm{H} 1272$ & 111.7 \\
\hline $\mathrm{C} 2-\mathrm{C} 27-\mathrm{H} 273$ & 111.3 & $\mathrm{C} 102-\mathrm{C} 127-\mathrm{H} 1273$ & 106.1 \\
\hline $\mathrm{H} 271-\mathrm{C} 27-\mathrm{H} 273$ & 109.4 & H1271-C127-H1273 & 112.4 \\
\hline $\mathrm{H} 272-\mathrm{C} 27-\mathrm{H} 273$ & 110.1 & $\mathrm{H} 1272-\mathrm{C} 127-\mathrm{H} 1273$ & 109.0 \\
\hline
\end{tabular}

